\title{
STABILITY AND CONTROL OF SUPERCRITICAL HELIUM FLOW IN THE LHC CIRCUITS
}

\author{
E. Hatchadourian
}

\begin{abstract}
The circulating particle beams of the Large Hadron Collider (LHC) will induce dynamic heat loads into the cryogenic system. Beam screens, maintained at a temperature between $5 \mathrm{~K}$ and $20 \mathrm{~K}$ by weakly supercritical helium -in order to avoid-two phase flow- are inserted inside the magnet cold bore to intercept most of these heat loads. Evidence has been presented in experimental and theoretical work that the main type of dynamic instability in long channels is that caused by the propagation of density waves due to multiple regenerative feedback. Oscillations are typically observed in circuits operating with low flow rate and/or high energy input. The study of the system behaviour under different operating cases permits assessment of the time constant of the system as well as its temperature-control parameters. A part of this work also concerns the study of flow stability in the other LHC cryogenic circuits working with supercritical helium.
\end{abstract}

LHC Division

Presented at the 1999 Cryogenic Engineering and International Cryogenic Materials Conference

(CEC-ICMC'99), 12-16 July 1999, Montreal, Canada

\author{
Administrative Secretariat \\ LHC Division \\ CERN \\ CH - 1211 Geneva 23 \\ Switzerland \\ Geneva, 1 December 1999
}




\title{
STABILITY AND CONTROL OF SUPERCRITICAL HELIUM FLOW IN THE LHC CIRCUITS
}

\author{
E. Hatchadourian \\ LHC Division, CERN \\ 1211 Geneva 23, Switzerland.
}

\begin{abstract}
The circulating particle beams of the Large Hadron Collider (LHC) will induce dynamic heat loads into the cryogenic system. Beam screens, maintained at a temperature between $5 \mathrm{~K}$ and $20 \mathrm{~K}$ by weakly supercritical helium -in order to avoid-two phase floware inserted inside the magnet cold bore to intercept most of these heat loads. Evidence has been presented in experimental and theoretical work that the main type of dynamic instability in long channels is that caused by the propagation of density waves due to multiple regenerative feedback. Oscillations are typically observed in circuits operating with low flow rate and/or high energy input. The study of the system behaviour under different operating cases permits assessment of the time constant of the system as well as its temperature-control parameters. A part of this work also concerns the study of flow stability in the other LHC cryogenic circuits working with supercritical helium.
\end{abstract}

\section{INTRODUCTION}

The design of the beam screen (figure 1) is based on a $1 \mathrm{~mm}$-thick stainless steel tube coated on its inner surface with $50 \mu \mathrm{m}$ copper ${ }^{1}$. Weakly supercritical helium, flowing in two stainless steel tubes of $3.7 \mathrm{~mm}$ inner diameter running on each side of the beam screen, maintain its temperature below $20 \mathrm{~K}$, while intercepting the heat loads deposited by synchrotron radiation, resistive dissipation of beam image currents, and impact of photoelectrons resonantly accelerated by the circulating proton bunches ${ }^{2}$. These beam-induced heat loads, which strongly depend on the intensity, amount to $0.46 \mathrm{~W} / \mathrm{m}$ and $1.13 \mathrm{~W} / \mathrm{m}$ respectively in so-called "nominal" and "ultimate" operating conditions ${ }^{3}$. After discussing the flow stability, we will analyse the control of the exit temperature in order to determine the parameters that are significant to the design of automatic control systems. The results of theoretical work has been validated on a full-scale test section which reproduces one of the cooling tubes.

\section{HYDRAULIC STABILITY}

Analytical approach. In order to study fluid behaviour through different LHC cryogenic lines, a mathematical investigation and experimental tests have been performed. Oscillations of a hydrodynamic system require a mechanism for temporary storage and release of some form of energy. 


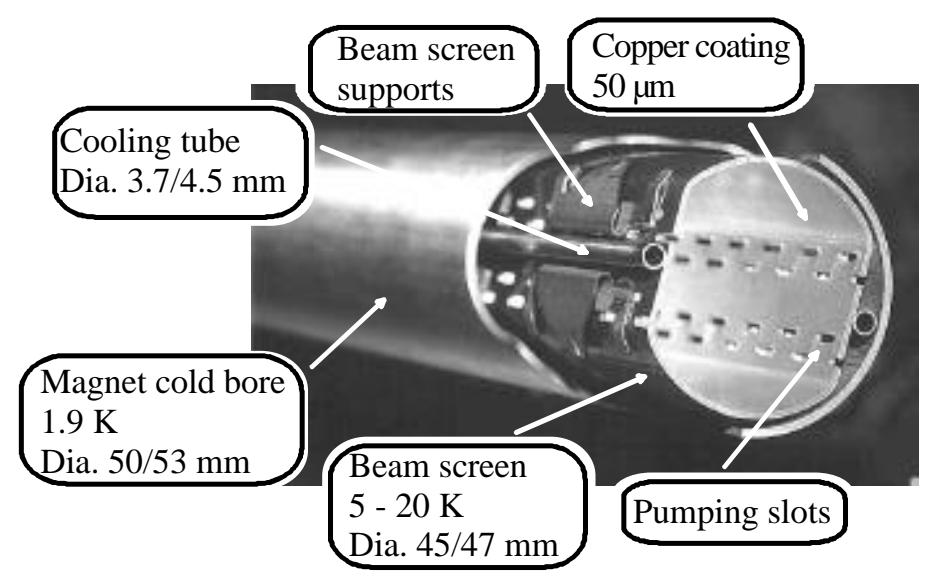

Figure 1. A view of the LHC beam screen.

In the case of constant heat load, at high flow rates little energy is stored and its amount increases with decreasing flow. The physical phenomenon can be described as follows : an inlet flow fluctuation creates an enthalpy perturbation in the high-density region, which travels with the flow along the channel. When this perturbation reaches the "change-ofdensity" boundary it is partly transmitted to the lower-density region. With correct timing, the perturbation can acquire appropriate phase and become self-sustained. The analysis is based on the assumption of an oscillatory inlet flow ${ }^{4}$, and consists in writing and linearising the fundamental equations -the conservation of mass, energy and momentum- describing the fluid state. Other assumptions are made in order to produce a simple stability map that can be used for different cases :

- the equation of state is approximated by two regions ; the first (quasi-liquid) representing the subcooled liquid fluid has a constant density, the second (quasi-gas) is modelled as ideal gas. This equation of state is plotted in figure 2 for 3 bar, the normal operating pressure of the cooling tube. The dependence of the friction factor ${ }^{4}$ on enthalpy (given by the Poiseuille and the Moody equations) is also shown in figure 2 at the same pressure. Exact properties for different operating pressures -between 2.4 and 3.6 bar- are represented by the cross-hatched area,

- the incompressible flow is assumed to be one-dimensional with uniform section along the heater line. The effect of diverse geometrical parameters (upstream and downstream adiabatic lengths, singularities) has been considered too,

- the thermal properties of the wall are not taken into account. The characteristic time due to thermal diffusivity in the tube wall is negligible in comparison with the period of the oscillations.

Stability criterion. The effect of a small perturbation of inlet velocity on the different regions (upstream length, quasi-liquid, quasi-gas, downstream length, singularities) can be found out from the linearised relations for conservation of mass and energy. Finally, the variation of the overall pressure drop (friction, acceleration and gravity terms in each region) is determined by integration of the linearised momentum relation.

The behaviour of an inlet velocity perturbation $\delta u$ is determined by a dynamic system analysis in which the input signal is the perturbation in pressure drop over the different regions, and the output signal is the inlet velocity perturbation. The arbitrary choice of the forward transfer function (the response of inlet velocity to upstream pressure drop) and the feedback transfer function (the response of the heated tube and downstream pressure drop to inlet conditions) determines the hydrodynamic closed loop system, which is analysed using the standard Nyquist criterion ${ }^{5}$. When the real part of the denominator of the closed loop transfer function F (1) is negative and its imaginary part is zero, then the system becomes oscillatory.

$$
\mathrm{F}=\left(\frac{\delta \Delta \mathrm{P}_{\text {upstream }}}{\delta \mathrm{u}}+\frac{\delta \Delta \mathrm{P}_{\text {quasi-liquid }}}{\delta \mathrm{u}}+\frac{\delta \Delta \mathrm{P}_{\text {quasi-gas }}}{\delta \mathrm{u}}+\frac{\delta \Delta \mathrm{P}_{\text {downstream }}}{\delta \mathrm{u}}\right)^{-1}
$$




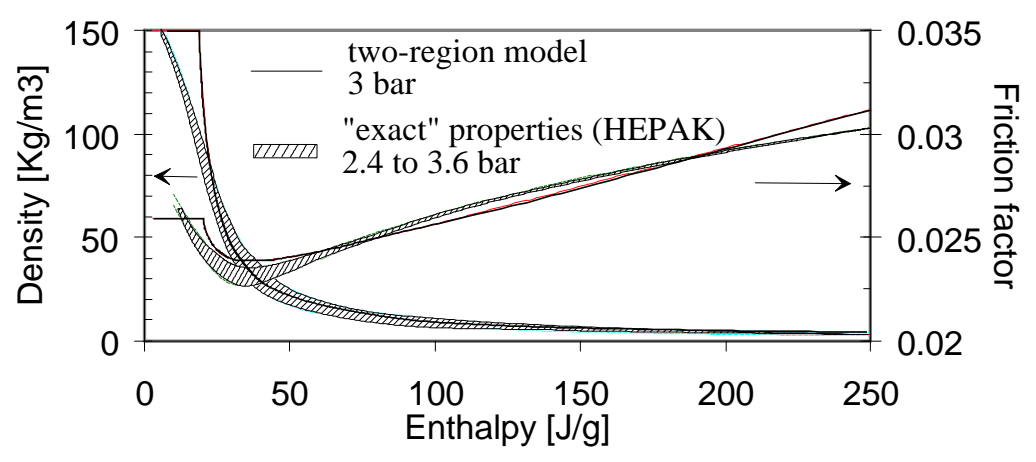

Figure 2. Density and frictional pressure drop coefficient versus enthalpy.

To account for the specific hydrodynamic characteristics of the different regions, it is necessary to treat separately the perturbation in each region.

Application to beam screen cooling tubes. From the above analysis, we expected oscillations for a wide range of operating conditions : inlet subcooling, low pressure, heat input, low flow rate and exit restriction. The inlet subcooling is defined by the enthalpy difference in the quasi-liquid region from inlet to the transition point. Figure 3 is a typical plot of the periods measured at the threshold of the stability versus heat input. The measured period of the oscillations ranged from 50 to 400 seconds, increasing continuously with subcooling.

A stability map -reduced inlet velocity (2) versus inlet subcooling enthalpy- for different upstream and downstream tube length and diameter is shown in figure 4 . The notation in equation (2) is as follows : $\mathrm{u}$ is the dimensional inlet fluid velocity, $\mathrm{L}$ is the heated length, $\mathrm{Q}$ is the total heat input , $\mathrm{v}$ is the ideal gas specific volume, $\mathrm{h}$ is the enthalpy and $\alpha$ is the expansion coefficient at constant pressure.

$$
\mathrm{u}_{\text {reduced }}=\frac{\mathrm{u}}{\mathrm{LQ}} \frac{1}{\alpha}, \quad \text { where } \alpha=\left.\frac{1}{\mathrm{v}} \frac{\mathrm{dv}}{\mathrm{dh}}\right|_{\mathrm{P}}
$$

Above the limit line, the flow is stable ; below its the flow is unstable. In figure 4a we can see the effect of unheated additional lengths (destabilising effect when downstream length increases). The length ratio is respectively 0.5 for downstream and 5 for upstream. Figure $4 \mathrm{~b}$ shows the result of varying the unheated tube diameters (with 0.2 fractional unheated length and with a same 0.5 diameter ratio for the two limit lines). When the diameter of the

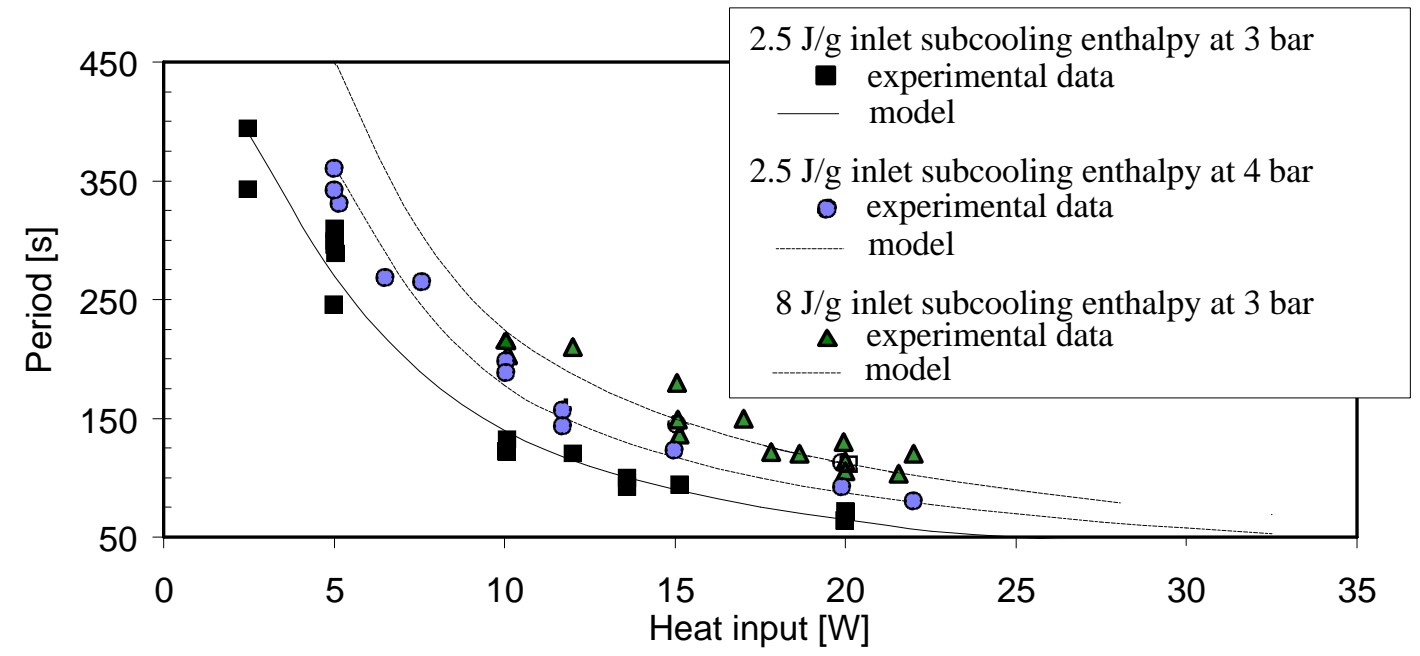

Figure 3. Period of oscillations versus heat input at the stability boundary. 

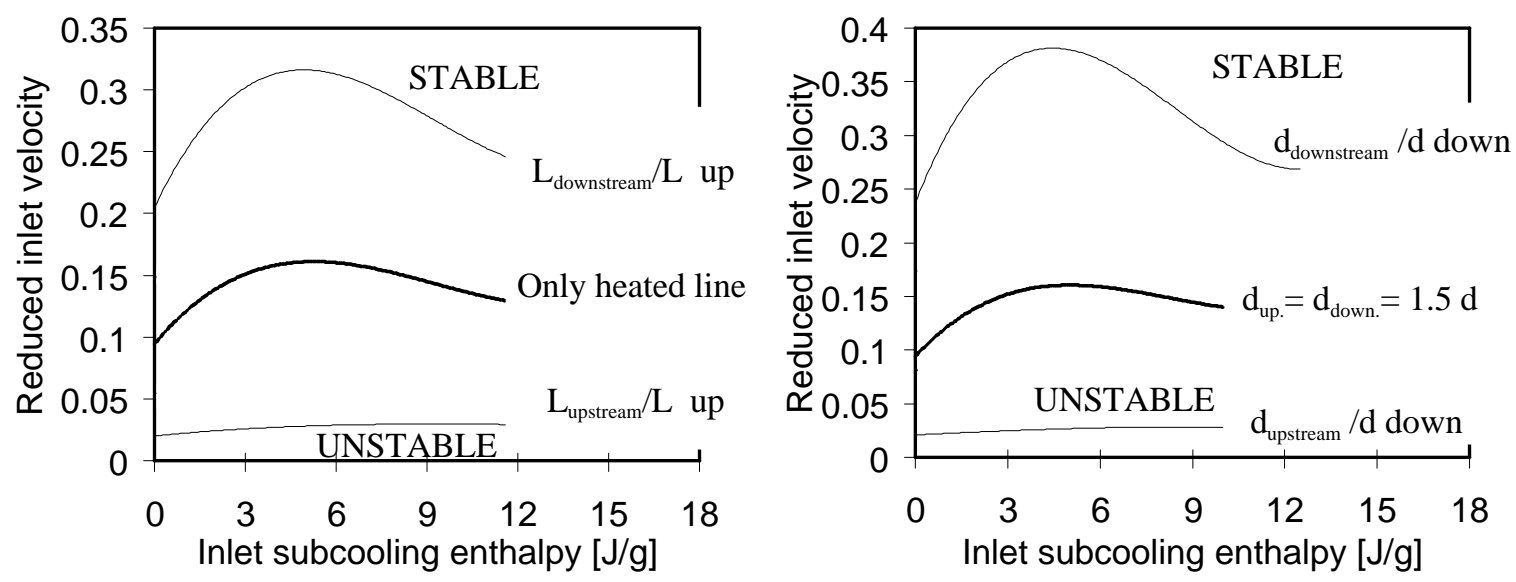

Figure 4. Stability boundaries for (a) different unheated tube length L and (b) unheated tube diameter d.

upstream or downstream lengths exceed 1.5 times that of the heated length, there is no effect on the stability diagram. At low subcooling an increase of subcooling renders the system less stable and at high subcooling, an increase of subcooling renders the system more stable.

Application to other LHC lines. While line C' (15 mm diameter) runs over the $53 \mathrm{~m}$ of a half cell, where it intercepts heat on the magnet supports before feeding the beam screen cooling tubes, line C (100 mm diameter) supplies supercritical helium over the 3.3 $\mathrm{km}$ length of an LHC sector. The cooling scheme for these 3 lines is shown in figure 5 . No density oscillations are expected in the supply header (line $\mathrm{C}$ ) because of the small heat loads deposited (principally radiation between $77 \mathrm{~K}$ and $4.6 \mathrm{~K}$ ) and the high mass flow rate (varying from $180 \mathrm{~g} / \mathrm{s}$ at the beginning of the sector down to $20 \mathrm{~g} / \mathrm{s}$ at the end).

Concerning line C', each of the 11 supports deposits $1.36 \mathrm{~W}$ locally, over a length of $30 \mathrm{~cm}$ (there are 2 supports on the quadrupole cryostat and 3 supports on each dipole cryostat). As long as the flow in the line remains in the pseudo-liquid domain, no oscillation is expected over the short length of the supports. If the mass flow rate decreases to less then $0.15 \mathrm{~g} / \mathrm{s}$ with an operating pressure close to the critical pressure, the system could become unstable : this case occurs if the control valve at the end of the line is maintained at a set point of $20 \mathrm{~K}$ with very small heating deposited on the beam screen line. Table 1 summarises the different supercritical lines. It should be noted that the heat input brought by the supports increases the inlet beam screen temperature and thus reduces the possibility of density oscillations.

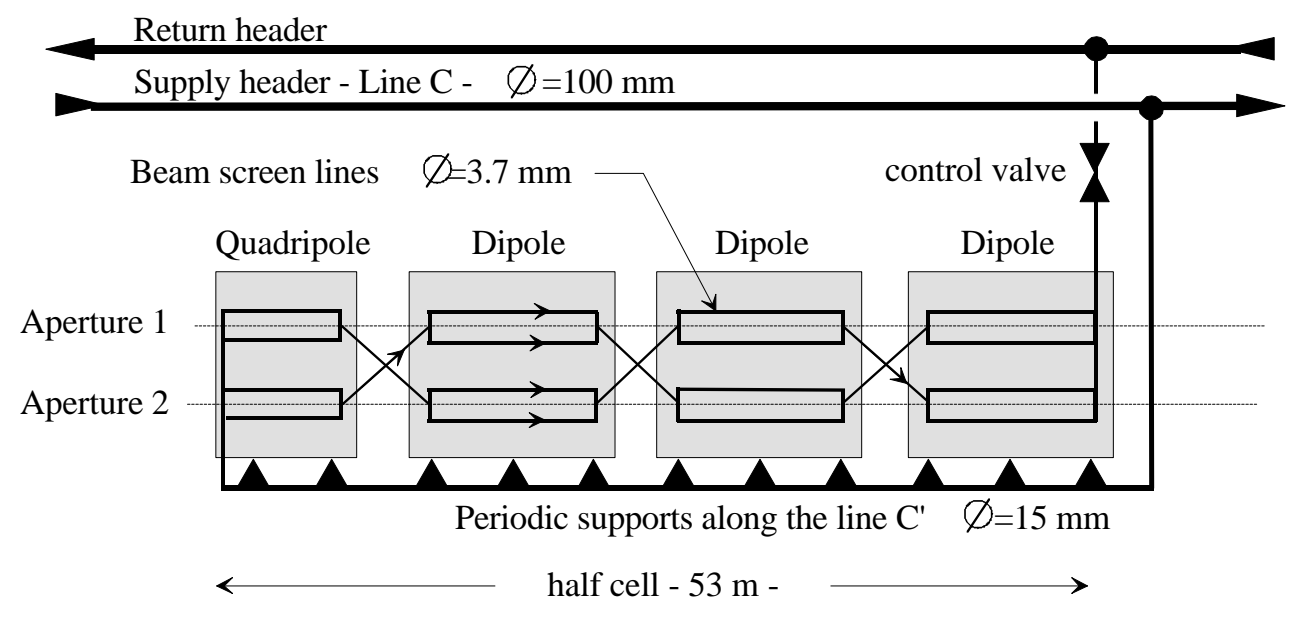

Figure 5. Supercritical helium circuits in the LHC. 
Table 1 Characteristics of the supercritical helium lines in the LHC.

\begin{tabular}{cccccc}
\hline Lines & $\begin{array}{c}\text { Length } \\
(\mathrm{m})\end{array}$ & $\begin{array}{c}\text { Diameter } \\
(\mathrm{mm})\end{array}$ & Heat load & $\begin{array}{c}\text { Density } \\
\text { oscillations }\end{array}$ \\
\hline $\mathrm{C}$ & 3300 & 100 & $0.018 \mathrm{~W} / \mathrm{m}$ & distributed & None \\
C' & 53 & 15 & $1.360 \mathrm{~W} /$ support & local & Possible \\
Beam Screen & 53 & 3.7 & nominal $: 0.458 \mathrm{~W} / \mathrm{m}$ & distributed & Possible \\
\hline
\end{tabular}

\section{OUTLET TEMPERATURE CONTROL}

The objective is to maintain the outlet temperature of the process fluid at its desired temperature set point $(20 \mathrm{~K})$ in the presence of variations of the process fluid flow or applied heating.

Transfer function. The feedback control scheme works as follows : the outlet temperature or controlled variable is measured with a sensor and transmitter that generates a signal $\mathrm{T}(\mathrm{mA})$ proportional to the temperature. The measurement is sent to a controller where it is compared to the set point. The signal difference between the measurement and the set point generates a controller output signal which is then converted and applied to the actuator of the control valve. The flow is then a function of the valve position. Variations in outlet temperature are measured by the sensor-transmitter and sent to the controller causing the controller output signal to vary. This in turn causes the control valve position and then the flow to vary. The block diagram for the feedback control loop is shown in figure 6 . The symbols in this figure are :

$\mathrm{E}$ is the error signal, $\mathrm{mA}$

$\mathrm{F}_{\mathrm{c}}$ is the controller transfer function, $\mathrm{mA} / \mathrm{mA}$

$F_{v}$ is the control valve transfer function, $(\mathrm{kg} / \mathrm{s}) / \mathrm{mA}$

$\mathrm{F}_{\mathrm{p}}$ is the process transfer function, $\mathrm{K} /(\mathrm{kg} / \mathrm{s})$

$\mathrm{F}_{\mathrm{d}}$ is the disturbance transfer function, $\mathrm{K} /(\mathrm{kg} / \mathrm{s})$

$\mathrm{H}$ is the sensor-transmitter transfer function, $\mathrm{mA} / \mathrm{K}$

$\mathrm{K}$ is the scale factor for the temperature set point, $\mathrm{mA} / \mathrm{K}$

We can determine the closed-loop transfer function (3) :

$$
\mathrm{T}_{\text {out }}=\frac{\mathrm{KF}_{\mathrm{c}} \mathrm{F}_{\mathrm{v}} \mathrm{F}_{\mathrm{p}}}{1+\mathrm{HF}_{\mathrm{c}} \mathrm{F}_{\mathrm{v}} \mathrm{F}_{\mathrm{p}}} \times \mathrm{T}_{\text {control }}+\frac{\mathrm{F}_{\mathrm{d}}}{1+\mathrm{HF}_{\mathrm{c}} \mathrm{F}_{\mathrm{v}} \mathrm{F}_{\mathrm{p}}} \times \text { Disturbance }
$$

In order to adjust the controller parameter (Proportional-Integral type) to the characteristics of the rest of the components in the loop, we need to have access to the process steady-gain $F_{\text {gain }}$, the effective process dead time $t_{0}$ and the effective process time constants $\tau$ of the open-loop transfer function $\mathrm{F}$ of the equivalent block diagram $\left(\mathrm{F}=\mathrm{F}_{\mathrm{v}} \cdot \mathrm{F}_{\mathrm{p}} \cdot \mathrm{H}\right)$. The procedure used is to work in open loop and apply a step change in the controller output signal. The process can then be characterised using a first-order dead-time model (4) :

$$
\mathrm{F}=\frac{\mathrm{F}_{\text {gain }} \mathrm{e}^{-\mathrm{t}_{0} \mathrm{~s}}}{1+\tau \mathrm{s}}
$$

The PI controller settings are then calculated using the Ziegler-Nichols tuning relations ${ }^{6}$. A typical test plot is sketched in Figure 7. The experimental data are compared with the first order model and with the transfer function of the density model described above (using inverse Laplace transform for a step input condition). 


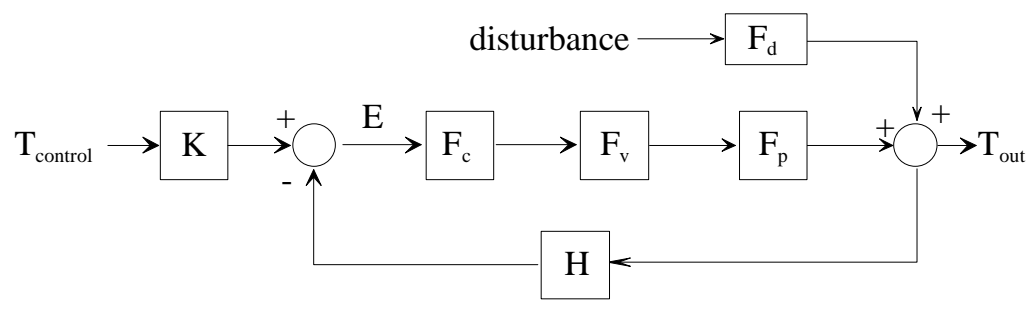

Figure 6. Block diagram for control loop of beam screen outlet temperature

Experimental results. The experiments for investigating the exit control temperature were of two classes : a heat load gradually increasing with time $-0.25 \mathrm{~W}$ per minute- up to "ultimate" LHC conditions, and heat load steps to simulate the "nominal" and "no beam" conditions (figure 8a). The controller settings have been obtained with the method described above. The operating range in these experiments was :

- inlet pressure between 2.5 and 4 bar with pressure drop less than 100 mbar,

- mass flow rate ranging from 0.1 to $0.3 \mathrm{~g} / \mathrm{s}$,

- a $3.75 \mathrm{~W}$ inlet heat load simulated the entry heat input for one cooling tube, which permitted an inlet temperature of the beam screen tube between 5.3 and $6.4 \mathrm{~K}$ (depending on pressure),

- the automatic control starts only when the temperature reaches the set point condition

$(20 \mathrm{~K})$ because of lower limitation in control valve position.

In accordance with the analysis, the destabilising effect of the pressure decrease could be observed (figure 8). The amplitude of temperature oscillations decrease with increasing pressure, whereas the period of oscillation increases. The period ranges from $80 \mathrm{~s}$ to $120 \mathrm{~s}$ close to "ultimate" heat input and between $120 \mathrm{~s}$ to $210 \mathrm{~s}$ for "nominal" heat input. The result also shows that the controller exit valve reacts correctly in case of oscillations.

The smoothing effect of azimuthal thermal conduction in the stainless-steel wall of the beam screen can be characterised by a time constant $\tau_{\text {screen }}(5)$. In view of symmetry -figure 9a- only a quarter of the beam screen perimeter is considered. The effect of the conduction is weakest at the furthest points from the cooling tubes.

$$
\tau_{\text {screen }}=\frac{\rho \mathrm{cl}^{2}}{\mathrm{k}}
$$

In this formula $\mathrm{k}$ is the conductivity, $\rho$ the density and $\mathrm{c}$ the specific heat of the beam screen stainless steel. In the range of temperatures encountered in the beam screen, this

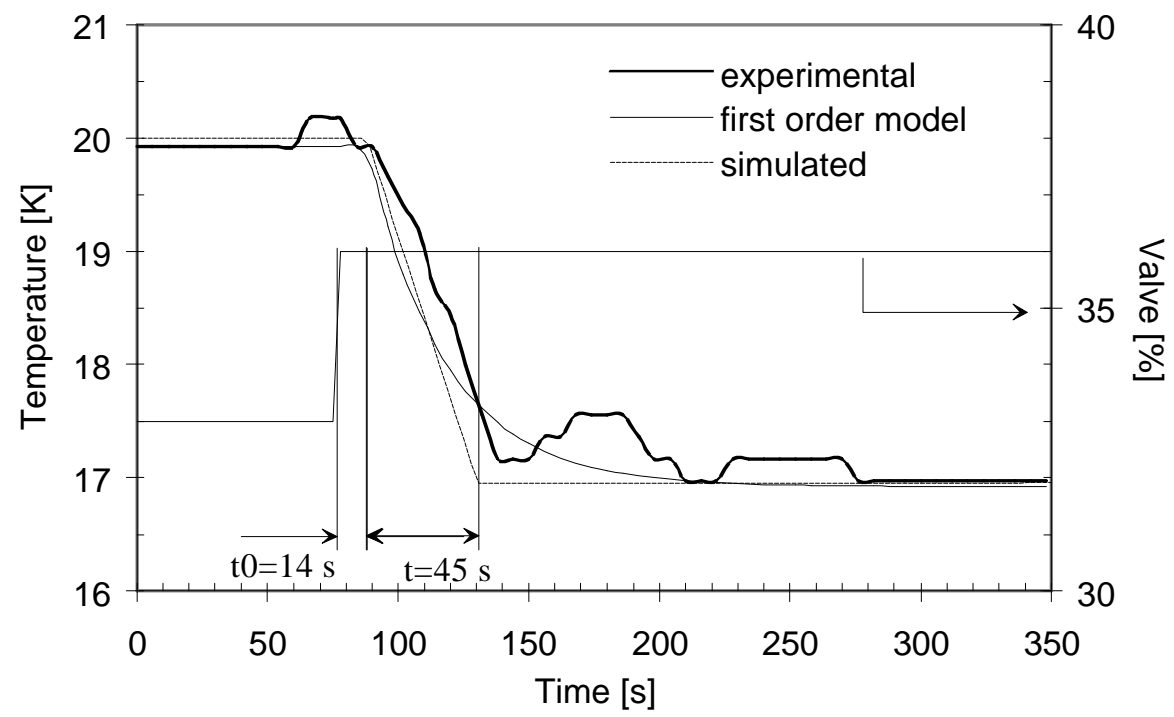

Figure 7. Open loop step response 


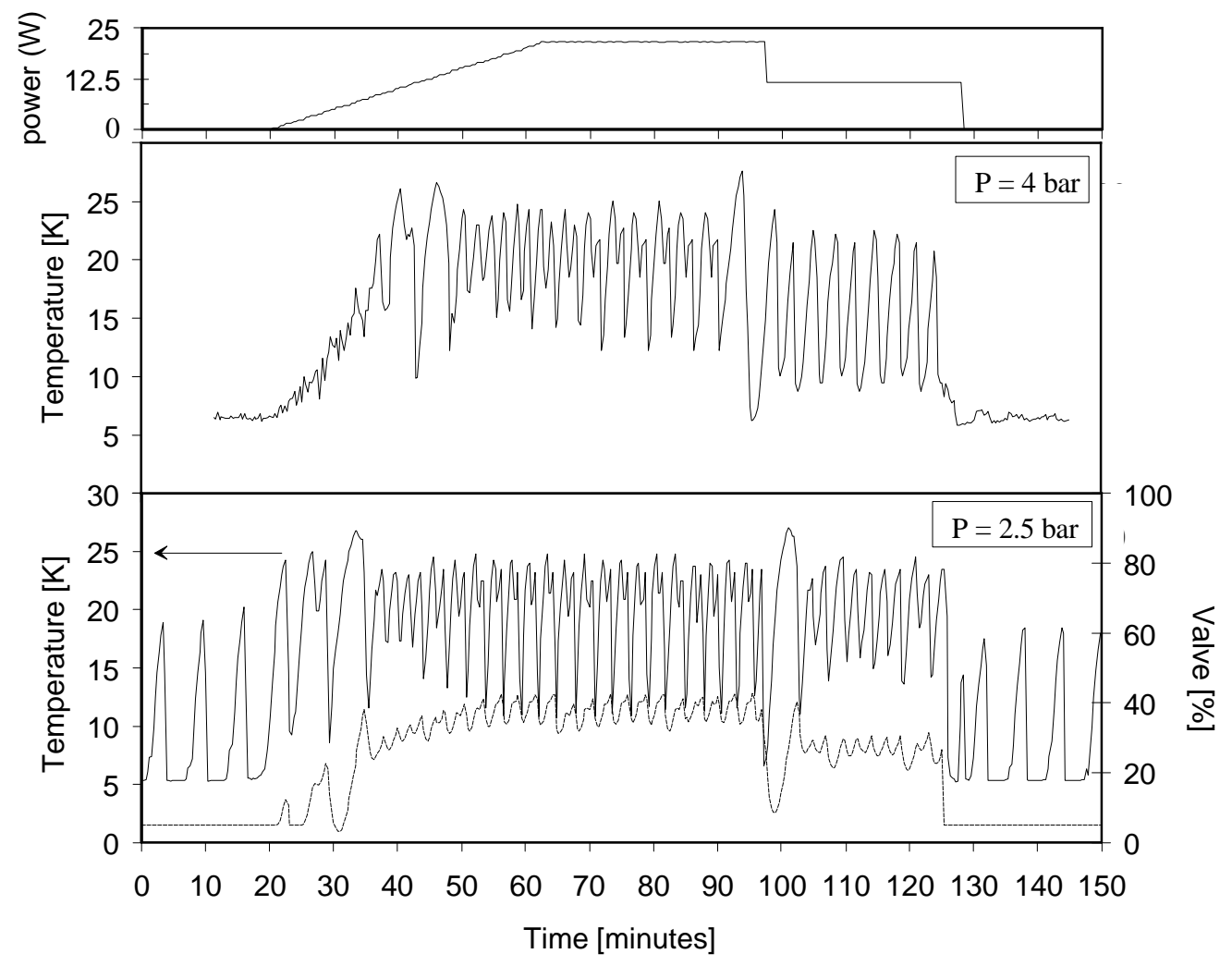

Figure 8a, b, c. Experimental exit temperature control

time constant (figure $9 \mathrm{~b}$ ) -for $\mathrm{l}=36 \mathrm{~mm}$ - shows little variation around 80 to 90 seconds. Above $20 \mathrm{~K}, \tau_{\text {screen }}$ increases due to the rise in specific heat of stainless steel ${ }^{7}$. We can conclude that the beam screen has a smoothing effect for the control of the exit temperature under oscillating flow conditions, for periods of oscillation below $\tau_{\text {screen }}$ such as encountered in "ultimate" condition. For periods higher than $\tau_{\text {screen, a numerical implicit }}$ investigation of the azimuthal conduction through the beam screen has led us to conclude that only less then $30 \%$ of the amplitude is propagated. A typical map showing the propagation of a temperature oscillation over length is represented in figure 10. Isotemperature lines are shown.
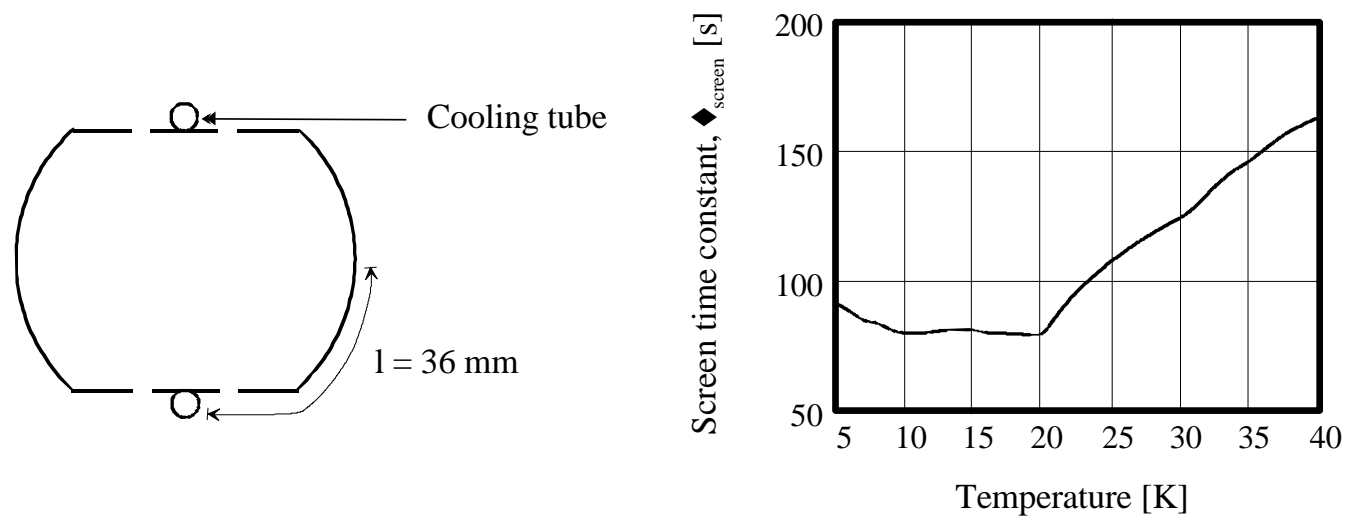

Figure 9. Transverse cross-section of beam screen (a) and screen time constant time versus temperature (b). 


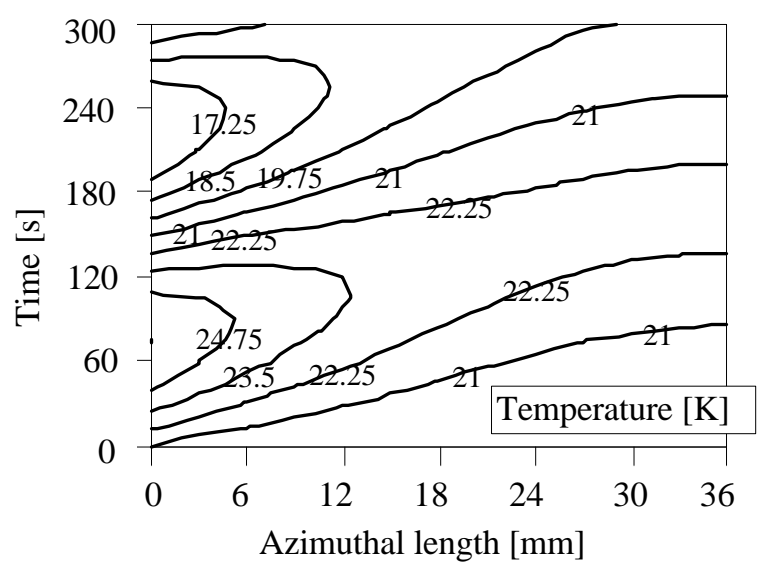

Figure 10. Azimuthal temperature propagation of a temperature oscillation with a period of 300 seconds and an amplitude of $10 \mathrm{~K}$ around $20 \mathrm{~K}$.

\section{CONCLUSION}

Theory and experiment have both established the risk of instability in supercritical helium flow. Some effects are complex like an increase of subcooling which may have a positive or negative effect depending on the inlet conditions. The influence of geometry on the driving and damping terms for oscillations has also been shown. Series of experiments made at different pressures have permitted testing the exit temperature control under stable and oscillatory conditions. A simple study of the beam screen azimuthal conduction has permitted determination of the smoothing effect.

\section{ACKNOWLEDGMENTS}

The author wishes to acknowledge the guidance of P. Lebrun, L. Tavian (CERN), and L. Puech (CNRS). Thanks also go to A. Bézaguet and his team (CERN) for their help with the experimental setup.

\section{REFERENCES}

1. P. Cruikshank and al. "Mechanical Design Aspects of the LHC Beam Screen", CERN LHC Project Report 128, (1997).

2. V. Baglin and al. "Beam-Induced Electron Cloud in the LHC and possible Remedies", CERN LHC Project Report 188, (1998).

3. E. Hatchadourian, Ph. Lebrun, L. Tavian. "Supercritical Helium Cooling of the LHC Beam Screens", Proc. ICEC17, IOP, 793 (1998).

4. R.C. Martinelli, D.B. Nelson. "Pressure Drop during Forced-Circulation Boiling of Water", Transactions of the ASME. 8:695 (1948).

5. N. Zuber. "An Analysis of Thermally Induced Flow Oscillations", Report NAS8-11422 (1966).

6. K. Ogata. "Modern Control Engineering", Prentice Hall, New Jersey, (1997).

7. H.M. Rosenberg. "Low Temperature Solid State Physics", Oxford, (1963). 